Psychotherapeut 2022 $67: 176-184$ https://doi.org/10.1007/s00278-021-00551-0 Angenommen: 11. Oktober 2021 Online publiziert: 5 . November 2021 (c) Der/die Autor(en) 2021

\section{Wartezeiten auf einen Psychotherapieplatz vor und nach der Psychotherapiestrukturreform}

\author{
Susanne Singer ${ }^{1}$. Lena Maier ${ }^{1} \cdot$ Anke Paserat $^{2} \cdot$ Klaus Lang $^{3} \cdot$ Bernhild Wirp $^{4}$. \\ Jörg Kobes ${ }^{5} \cdot$ Udo Porsch $^{6} \cdot$ Martina Mittag $^{7} \cdot$ Gerrit Toenges $^{8} \cdot$ Deborah Engesser $^{1}$ \\ ${ }^{1}$ Abteilung Epidemiologie und Versorgungsforschung, Institut für Medizinische Biometrie, Epidemiologie \\ und Informatik (IMBEI), Universitätsmedizin, Johannes Gutenberg-Universität, Mainz, Deutschland \\ ${ }^{2}$ Psychotherapeutische Praxis, Dresden, Deutschland \\ ${ }^{3}$ Psychotherapeutische Praxis, München, Deutschland \\ ${ }^{4}$ Psychotherapeutische Praxis, Braunschweig, Deutschland \\ ${ }^{5}$ Psychotherapeutische Praxis, Jena, Deutschland \\ ${ }^{6}$ Mainzer Psychoanalytisches Institut, Mainz, Deutschland \\ ${ }^{7}$ Psychotherapeutische Praxis, Stuttgart, Deutschland \\ ${ }^{8}$ Abteilung Biometrie, Institut für Medizinische Biometrie, Epidemiologie und Informatik (IMBEI), \\ Universitätsmedizin Mainz, Mainz, Deutschland
}

\section{Zusatzmaterial online}

Zusätzliche Informationen sind in der Online-Version dieses Artikels (https:// doi.org/10.1007/s00278-021-00551-0) enthalten.

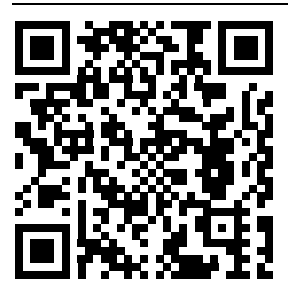

QR-Code scannen \& Beitrag online lesen

\title{
Zusammenfassung
}

Hintergrund und Fragestellung: Die Psychotherapiestrukturreform im Jahr 2017 verfolgte u. a. das Ziel, die Wartezeiten auf Psychotherapien zu verringern. Diese Studie ging der Frage nach, ob dieses Ziel erreicht wurde.

Methoden: Psychotherapiepraxen aus 7 Bundesländern entnahmen ihren Akten die Daten für Erstkontakt, Erstgespräch und Behandlungsbeginn. Der Vergleich der Wartezeiten auf ein Erstgespräch und auf den Beginn der Behandlung vor vs. nach der Reform erfolgte mithilfe von Kaplan-Meier-Kurven und stratifizierten Log-Rank-Tests. Ergebnis: Akten von 1548 Patienten aus 9 Praxen wurden ausgewertet. Die durchschnittliche Wartezeit auf ein Erstgespräch lag sowohl vor als auch nach der Reform bei 3 Wochen $(p=0,20)$. Die Zeit zwischen der Anmeldung und dem Behandlungsbeginn erhöhte sich von durchschnittlich 18 auf 20 Wochen, wobei nach der Reform v. a. die sehr kurzen Wartezeiten ( $<10$ Wochen) abnahmen $(p=0,0004)$. Diskussion: Die in den Praxen dokumentierten Daten liefern keine Anhaltspunkte dafür, dass sich die Wartezeit auf Erstgespräche bei Psychotherapeuten verkürzt hat. Das Warten auf einen Psychotherapieplatz scheint insgesamt etwas länger geworden zu sein, sodass in dieser Hinsicht keine Verbesserung der Versorgung durch die Reform festgestellt werden konnte.

\section{Schlüsselwörter}

Erstgespräch · Behandlungsbeginn · Behandlungsverzögerung · Versorgungsstrukturen · Inanspruchnahme der Gesundheitsversorgung

Das Warten auf eine fachgerechte Behandlung ist für Menschen mit psychischen Erkrankungen sehr belastend (Bridler et al. 2013; Wiegand et al. 2020; Williams et al. 2008). Aus diesem Grund bestand ein wichtiges Ziel der Reform der Psychotherapie-Richtlinie im Jahr 2017 darin, die Wartezeiten auf ein Erst- gespräch und den Beginn der Psychotherapie zu verkürzen (Gemeinsamer Bundesausschuss 2016, 2017; G-BA). Dies sollte durch die Einführung von Sprechstunden und Akutbehandlungen, die Vereinfachung des Antrags- und Gutachterverfahrens mit Genehmigungsfiktion sowie die Förderung von Gruppen- 
therapien erreicht werden. Ob sich die Wartezeiten durch die Psychotherapiestrukturreform tatsächlich verringerten, bleibt jedoch noch zu prüfen.

\section{Einleitung}

Die Bundespsychotherapeutenkammer (2018; BPtK) befragte im November 2017 insgesamt 9432 psychologische Psychotherapeuten und Kinder- und Jugendlichenpsychotherapeuten, wie lange Patienten bei ihnen auf Gespräche warten müssen. Diese schätzten, dass sich die Wartezeit auf Erstgespräche von 12,5 Wochen im Jahr 2011 auf 5,7 Wochen im November 2017 verringert hat; nur 8,5\% der Patienten müssten noch länger als 3 Monate warten. Die Wartezeit auf den Beginn einer Psychotherapie entsprechend der Richtlinien des G-BA habe sich jedoch um nur 3 Wochen verkürzt: von 23 Wochen im Jahr 2011 auf 20 Wochen im November 2017 (Bundespsychotherapeutenkammer 2018). Ähnliche lange Wartezeiten hatte schon eine Psychotherapeutenbefragung kurz nach dem Inkrafttreten des Psychotherapeutengesetzes 1999 ergeben (Zepf et al. 2003). Dass Patienten weniger lang auf ein Erstgespräch warten müssen, bedeutet also nicht, dass sie auch wesentlich schneller psychotherapeutisch behandelt werden können, denn dies ergibt sich womöglich v.a. aus den Kapazitäten der Psychotherapeuten. Je weniger Kassensitze pro Einwohner es in einer Region gibt, desto länger müssen Patienten auf eine Richtlinienpsychotherapie warten (Bundespsychotherapeutenkammer 2018). Darüber hinaus hängt die Wartezeit auch mit der Organisationsstruktur einer Praxis zusammen (Singer et al. 2017).

Obgleich die BPtK-Befragung wichtige Hinweise auf die Erfolge und Probleme der Strukturreform gab, sind ihre Ergebnisse nur begrenzt aussagekräftig, v.a. aus zwei Gründen: a) Ärztliche Psychotherapeuten wurden nicht befragt; diese erbringen aber einen Teil der psychotherapeutischen Versorgung (Gaebel et al. 2016; Kruse et al. 2013). b) Die Wartezeiten wurden nicht gemessen, sondern von den Psychotherapeuten geschätzt. Es blieb also zu klären, wie lang die tatsächlichen (dokumentierten) Wartezeiten sind. Deshalb förderte der Innovationsfonds des G-BA die vorliegen- de Studie, in der Daten aus den Akten mehrerer Psychotherapiepraxen verschiedener Berufsgruppen extrahiert wurden, um folgende Fragen zu beantworten:

1. Sind die Wartezeiten auf ein Erstgespräch in einer psychotherapeutischen Praxis nach der Psychotherapiestrukturreform kürzer geworden?

2. Sind die Wartezeiten auf den Beginn der Behandlung in einer psychotherapeutischen Praxis nach der Psychotherapiestrukturreform kürzer geworden?

Dazu wurden, basierend auf den Ergebnissen der BPtK-Befragung, folgende Hypothesen aufgestellt:

a) Die Wartezeit auf ein Erstgespräch hat sich durch die Reform im Durchschnitt reduziert.

b) Die Wartezeit bis zum Behandlungsbeginn hat sich im Durchschnitt nicht reduziert.

\section{Methode}

\section{Stichprobe}

Psychotherapeuten müssen die Zeit zwischen einem Erstkontakt (Anfrage der Patienten) und einem Erstgespräch regulär nicht dokumentieren, und die meisten tun dies auch nicht. Es ist daher nicht möglich, eine Zufallsstichprobe von Psychotherapeuten z.B. aus dem Register einer kassenärztlichen Vereinigung zu ziehen und diese dann um die Übermittlung ihrer Wartezeitdaten zu bitten. Deshalb wurde in einem Netzwerk niedergelassener Psychotherapeuten einer interdisziplinären Fachgesellschaft ${ }^{1}$ sowie bei Ausbildungsinstituten erfragt, wer seit mehreren Jahren Daten zur Wartezeit dokumentiert und archiviert. Insgesamt 8 Praxen und eine Ausbildungsambulanz meldeten sich (im Folgenden zusammenfassend als Praxen bezeichnet) und gaben an, diese Daten zur Verfügung zu haben. Die Praxen stammen aus verschiedenen Bundesländern, sowohl aus dem ländlichen als auch städtischen Raum, mit verschiedenen Praxisorganisationen und fachlicher Ausrichtung (• Tab. 1).

\footnotetext{
1 Arbeitsgemeinschaft Psychoonkologie der Deutschen Krebsgesellschaft.
}

\section{Fallzahlkalkulation}

Der Fallzahlkalkulation lag der in der BPtK-Studie (Bundespsychotherapeutenkammer 2018) ermittelte Unterschied von 20 vs. 23 Wochen Wartezeit zugrunde. Eine Standardabweichung (SD) wurde dort nicht berichtet. Liegt diese bei 10, kann der Unterschied von 20 vs. 23 bei einem a-Wert von 0,01 und einer Power von 0,80 mithilfe des Wilcoxon-Rangsummentests mit $n=331 /$ Gruppe (vor vs. nach der Reform) gegen den Zufall abgesichert werden. Bei einer SD von 15 und ansonsten gleichen Annahmen wären $n=744$ nötig, zusammen also 1488 Teilnehmende. Aufgrund der geclusterten Datenstruktur und der zu erwartenden Zensierungen wurde geplant, Wartezeitdaten von 1600 Patienten zu erfassen.

\section{Datenerfassung}

Die Praxen wurden gebeten, für jeweils 100 konsekutive Patienten in den Jahren vor und nach der Strukturreform (2016 und 2018) aus den Akten die folgenden Parameter zu extrahieren:

- Datum des Erstkontakts (telefonisch oder schriftlich),

- Datum des Erstgesprächs (probatorisches Gespräch oder Sprechstunde),

- Datum der letzten probatorischen Sitzung (bei Patienten nach der Reform auch das Datum der letzten Sprechstunde) und

- Datum des Behandlungsbeginns (Richtlinienpsychotherapie und/oder Akutbehandlung), falls es zu einer Behandlung kam.

Wenn weniger als 100 Patienten/Jahr die Praxis kontaktierten, konnten Daten aus weiteren Jahren hinzugenommen werden. Folgebehandlungen wurden ausgeschlossen. Es wurde ebenfalls extrahiert, wie viele probatorische Sitzungen bzw. Sprechstunden ein Patient erhalten hatte, ob eine Behandlung zustande gekommen war und, wenn nein, warum nicht. Ferner wurden Alter, Geschlecht, Bildungsgrad, die Art der Krankenversicherung und die F-Diagnose(n) gemäß der 10. Auflage der Internationalen statistischen Klassifikation der Krankheiten und verwandter Gesundheitsprobleme (ICD-10) erfasst. Die Daten wur- 
Tab. 1 Charakteristika der Psychotherapeuten/Psychotherapiepraxen $(n=9)$

\begin{tabular}{|c|c|c|}
\hline Merkmal & Ausprägung & $n$ \\
\hline \multirow[t]{2}{*}{ Geschlecht } & Männlich & 2 \\
\hline & Weiblich & 7 \\
\hline \multirow[t]{3}{*}{ Praxisform } & Einzelpraxis & 7 \\
\hline & Gemeinschaftspraxis & 0 \\
\hline & Andere Praxisform (z. B. Ausbildungsambulanz) & 2 \\
\hline \multirow[t]{3}{*}{ Kassensitz vor der Reform } & Kein Kassensitz & 1 \\
\hline & Halber Kassensitz & 3 \\
\hline & Voller Kassensitz & 5 \\
\hline \multirow{3}{*}{$\begin{array}{l}\text { Kassensitz nach der Re- } \\
\text { form }\end{array}$} & Kein Kassensitz & 1 \\
\hline & Halber Kassensitz & 4 \\
\hline & Voller Kassensitz & 4 \\
\hline \multirow[t]{3}{*}{ Berufa } & Arzt/Ärztin & 4 \\
\hline & Psychologe/Psychologin & 6 \\
\hline & $\begin{array}{l}\text { Anderer (z. B. Pädagoge/Pädagogin, Sozialpädagoge/ } \\
\text { Sozialpädagogin) }\end{array}$ & 1 \\
\hline \multirow{3}{*}{$\begin{array}{l}\text { Abrechnungs- } \\
\text { genehmigung }\end{array}$} & Tiefenpsychologisch fundierte Psychotherapie & 2 \\
\hline & Verhaltenstherapie & 5 \\
\hline & $\begin{array}{l}\text { Analytische Psychotherapie und tiefenpsychologisch fundier- } \\
\text { te Psychotherapie }\end{array}$ & 2 \\
\hline \multirow[t]{3}{*}{ Altersschwerpunkt } & Kinder und Jugendliche & 0 \\
\hline & Erwachsene & 7 \\
\hline & Beide Gruppen & 2 \\
\hline Ergänzungsqualifikation & Kinder- und Jugendlichenpsychotherapie & 2 \\
\hline $\begin{array}{l}\text { Psychoonkologischer } \\
\text { Schwerpunkt }\end{array}$ & Ja & 7 \\
\hline \multirow{4}{*}{$\begin{array}{l}\text { Kreistyp bei Bedarfspla- } \\
\text { nung }\end{array}$} & Typ 1 & 7 \\
\hline & Typ 2 & 0 \\
\hline & Typ 3 & 1 \\
\hline & Typ 4 & 1 \\
\hline
\end{tabular}

den pseudonymisiert an die Studienzentrale in Mainz geschickt.

Für jede Praxis wurde erhoben, ob es sich um eine Einzel- oder Gemeinschaftspraxis handelt, des Weiteren der Kreistyp (im Sinne der Kassensitzbedarfsplanung), die Gemeindegröße, die Art des Kassensitzes sowie die Berufsgruppe und das angebotene Therapieverfahren des Therapeuten.

\section{Statistische Analyse}

Die Dauer zwischen dem Tag der Anmeldung (Erstkontakt) bis zum Tag des Erstgesprächs (Wartezeit auf Erstgespräch) sowie die Dauer vom Tag der Anmeldung bis zum Beginn der Akutbehandlung oder Beginn der Richtlinienpsychotherapie (Wartezeit auf Behandlungsbeginn) in Tagen, Wochen und Monaten wurde berechnet.
Verglichen wurden die Wartezeiten von Patienten, deren Erstkontakt vor April 2017 stattfand (vor der Reform) mit denen ab April 2017 (nach der Reform). Für die beobachteten Wartezeiten wurden Mittelwert, SD, Median, Minimum und Maximum separat für vor und nach der Reform ermittelt.

Bei der statistischen Analyse wurde berücksichtigt, dass es sich um geclusterte Daten handelt, also um Wartezeiten von verschiedenen Patienten innerhalb von Praxen. Es wurde davon ausgegangen, dass sich die Wartezeiten innerhalb einer Praxis ähneln könnten. Deshalb wurden stratifizierte Log-Rank-Tests, mit den Praxen als Strata, durchgeführt.

Patienten, die ins Krankenhaus überwiesen wurden oder bei denen in dieser Praxis keine Behandlung möglich oder diese nicht indiziert war, wurden bezüglich der Wartezeit auf den Behandlungsbe- ginn anhand des Datums der Krankenhauseinweisung oder des Datums des letzten Gesprächs (letzte Probatorik oder letzte Sprechstunde) zensiert. Die Wartezeiten auf ein Erstgespräch sind nicht zensiert, da alle in den Akten dokumentierten Patienten ein Erstgespräch erhielten.

Da die Umsetzung der Reform weder mit Patienten- noch mit Praxismerkmalen zusammenhängt, kann keine Konfundierung vorliegen, weshalb nicht für entsprechende Merkmale adjustiert und deshalb auch keine regressionsanalytische Methode verwendet wurde.

Alle Berechnungen wurden aus Qualitätssicherungsgründen von zwei Wissenschaftlerinnen (S.S. und D.E.) unabhängig voneinander vorgenommen. Die Analysen erfolgten mithilfe der Statistiksoftware STATA, Version 15.1 (Fa. StataCorp, Texas).

\section{Ergebnisse}

\section{Stichprobencharakteristika}

Akten von insgesamt 1548 Patienten wurden ausgewertet (79 bis 250 Patienten/ Praxis). Diese waren zu 75\% weiblichen Geschlechts und in 10\% der Fälle minderjährig (Details: - Tab. 2). Es waren alle Bildungsniveaus vertreten, von keinerlei Schulabschluss (1\%) über Hauptschulabschluss (13\%) bis zu Abitur (34\%). Die häufigsten Diagnosen waren dem Kapitel F4 der ICD-10, also neurotische, Belastungsund somatoforme Störungen, gefolgt von affektiven Störungen (Kapitel F3) zuzuordnen. Es waren aber auch Patienten mit Intelligenzminderung (F7), Entwicklungsstörungen (F8) und organischen Störungen (F0) vertreten.

\section{Wartezeiten auf das Erstgespräch}

Insgesamt 755 Patienten hatten sich vor und 793 nach der Reform bei den Therapeuten angemeldet. Zwischen dem Erstkontakt und dem Erstgespräch vergingen vor der Reform im Durchschnitt 22,5 Tage, d.h. 3,2 Wochen (SD 3,9 Wochen, Median 2 Wochen, Spanne: 0 Tage bis 49 Wochen) und nach der Reform im Durchschnitt 22,2 Tage, also ebenfalls 3,2 Wochen (SD 3,9 Wochen, Median 2 Wochen, Spanne: 0 Tage bis 37 Wochen). Der nach 


\begin{tabular}{|c|c|c|c|}
\hline Merkmal & Ausprägung & $n$ & $\%$ \\
\hline \multirow[t]{3}{*}{ Geschlecht } & Männlich & 385 & 25 \\
\hline & Weiblich & 1162 & 75 \\
\hline & Divers & 1 & 0,1 \\
\hline \multirow[t]{2}{*}{ Alter } & $<18$ Jahre & 158 & 10 \\
\hline & Volljährig & 1390 & 90 \\
\hline \multirow[t]{8}{*}{ Bildung } & Kein Schulabschluss & 10 & 1 \\
\hline & Hauptschule & 195 & 13 \\
\hline & Realschule & 303 & 20 \\
\hline & Fachhochschulreife & 61 & 4 \\
\hline & Abitur & 533 & 34 \\
\hline & Anderer Schulabschluss & 12 & 1 \\
\hline & Noch Schüler oder noch nicht im schulfähigen Alter & 124 & 8 \\
\hline & Unbekannt & 310 & 20 \\
\hline \multirow{10}{*}{$\begin{array}{l}\text { F-Diagnosen } \\
\text { (mehrere pro } \\
\text { Person möglich) }\end{array}$} & F0 - Organische Störungen & 4 & 0,3 \\
\hline & $\begin{array}{l}\text { F1 - Psychische und Verhaltensstörungen durch psycho- } \\
\text { trope Substanzen }\end{array}$ & 25 & 2 \\
\hline & F2 - Schizophrenie, schizotype und wahnhafte Störungen & 8 & 1 \\
\hline & F3 - Affektive Störungen & 354 & 23 \\
\hline & $\begin{array}{l}\text { F4 - Neurotische, Belastungs- und somatoforme Störun- } \\
\text { gen }\end{array}$ & 1198 & 77 \\
\hline & $\begin{array}{l}\text { F5 - Verhaltensauffälligkeiten mit körperlichen Störungen } \\
\text { und Faktoren }\end{array}$ & 80 & 5 \\
\hline & F6 - Persönlichkeits- und Verhaltensstörungen & 89 & 6 \\
\hline & F7 - Intelligenzminderung & 3 & 0,2 \\
\hline & F8 - Entwicklungsstörungen & 3 & 0,2 \\
\hline & $\begin{array}{l}\text { F9 - Verhaltens- und emotionale Störungen mit Beginn in } \\
\text { der Kindheit und Jugend }\end{array}$ & 97 & 6 \\
\hline \multirow{4}{*}{$\begin{array}{l}\text { Kranken- } \\
\text { versicherung }\end{array}$} & Gesetzlich & 1337 & 86 \\
\hline & Privat & 125 & 8 \\
\hline & Beihilfe & 68 & 4 \\
\hline & Sonstiges & 18 & 1 \\
\hline
\end{tabular}

Praxen stratifizierte Log-Rank-Test ergab keine Hinweise auf Unterschiede in der Wartezeit auf ein Erstgespräch vor vs. nach der Reform $\left(X^{2} 1,7 ; p=0,20\right)$. Auch die Kaplan-Meier-Kurve macht deutlich, dass die Wartezeiten sich sehrähneln (• Abb. 1). Dasselbe Muster zeigt sich, wenn nur die gesetzlich Versicherten betrachtet werden (Zusatzmaterial online: Abb. 1).

Um zu prüfen, ob dieses Ergebnis womöglich daran lag, dass viele der Praxen einen psychoonkologischen Schwerpunkt haben, wurde eine Subgruppenanalyse für nur die Praxen ohne einen solchen Schwerpunkt durchgeführt. Hier betrugen die Wartezeiten 3,2 Wochen vor der Reform und 3,6 Wochen nach der Reform $(p=0,30)$.
Wartezeiten auf den Behandlungsbeginn

Nach dem Erstgespräch und ggf. weiteren Gesprächen kam es in 663 Fällen zu einer Behandlung in der Praxis (303 vor und 360 nach der Reform). Gründe, keine Behandlung zu beginnen, bestanden v.a. darin, dass keine Indikation für eine ambulante Psychotherapie gegeben war (24\%), dass die Patienten die Behandlung ablehnten (11\%), dass lediglich eine Krisenintervention nötig war (10\%), dass der Therapeut keine freien Kapazitäten hatte $(7 \%)$ oder dass andere Stellen (z. B. Therapeuten mit einem anderen Therapieverfahren oder Altersschwerpunkt) für die Behandlung geeigneter erschienen und der Patient dorthin verwiesen wurde $(6 \%$; $\mathbb{0}$ Tab. 3$)$.
Zwischen dem Erstkontakt und dem Behandlungsbeginn vergingen vor der Reform im Durchschnitt 126 Tage, also 17,9 Wochen (SD 12,6 Wochen, Median 15 Wochen, Spanne: 11 Tage bis 17 Monate), und nach der Reform im Durchschnitt 141 Tage, also 20,1 Wochen (SD 11,3 Wochen, Median 19 Wochen, Spanne: 4 Tage bis 2 Jahre). Wenn nach der Reform die Behandlung mit einer Akutbehandlung begonnen wurde, betrug die Wartezeit im Durchschnitt 14,5 Wochen, ( $n=101$, SD 7,4 Wochen). Wenn hingegen mit einer Richtlinientherapie begonnen wurde, betrug sie durchschnittlich 22,3 Wochen ( $n=259$, SD 11,8 Wochen).

Bei den Patienten, bei denen keine Behandlung in der Praxis zustande kam, vergingen zwischen dem Erstkontakt und dem letzten Gespräch bzw. der Krankenhauseinweisung vor der Reform im Durchschnitt 7,9 Wochen (SD 8,0 Wochen, Median 6 Wochen, Spanne: 0 Tage bis 13 Monate) und nach der Reform im Durchschnitt 8,3 Wochen (SD 10,2 Wochen, Median 5 Wochen, Spanne: 0 Tage bis 2 Jahre).

Der stratifizierte Log-Rank-Test gibt Hinweise auf überzufällige Unterschiede in der Wartezeit auf den Behandlungsbeginn vor und nach der Reform $\left(X^{2}=12,7\right.$; $p=0,0004)$. An der Kaplan-Meier-Kurve wird ersichtlich, dass die Wartezeiten auf den Behandlungsbeginn bis etwa 30 Wochen nach dem Erstkontakt vor der Reform kürzer waren; ab etwa diesem Zeitpunkt begann die Behandlung nach der Reform schneller (®Abb. 2). Dasselbe Muster findet sich, wenn nur die gesetzlich Versicherten betrachtet werden (Zusatzmaterial online: Abb. 2). Da die Kurven sich kreuzen, hat der Log-Rank-Test eine geringere Power, Unterschiede zwischen den Wartezeitverteilungen zu entdecken, aufgrund der hohen Fallzahlen bleibt der Unterschied trotzdem signifikant.

Werden die beobachteten Wartezeiten gruppiert, zeigt sich, dass sowohl die sehr kurzen $(<10$ Wochen) als auch die sehr langen Wartezeiten ( $>40$ Wochen) nach der Reform abnahmen (Zusatzmaterial online: Tab. 1). 


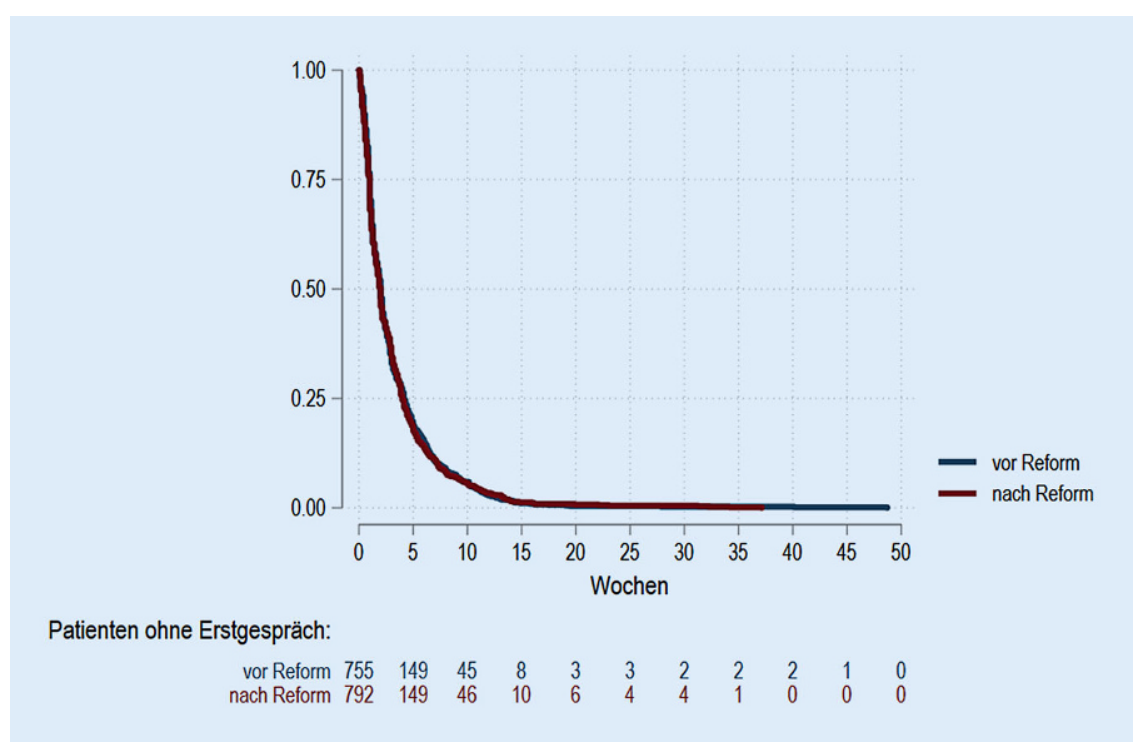

Abb. $1 \Delta$ Anteil der Patienten, die nach dem Erstkontakt (Anmeldung in der Praxis) noch kein Erstgespräch erhalten haben, im Verlauf der Zeit seit dem Erstkontakt

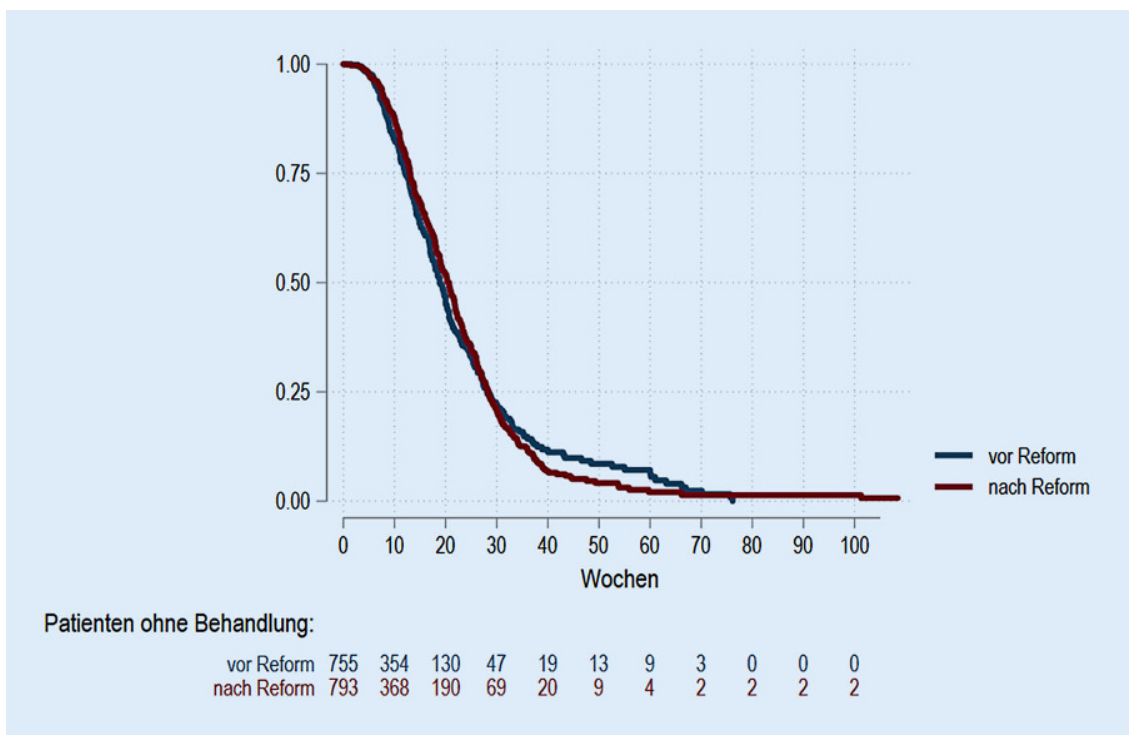

Abb. 2 \ Anteil der Patienten, die nach dem Erstkontakt (Anmeldung in der Praxis) noch keine Behandlung (entweder als Akutbehandlung oder als Richtlinienpsychotherapie) erhalten haben, im Verlauf der Zeit seit dem Erstkontakt. Patienten, die ins Krankenhaus überwiesen wurden oder bei denen keine Behandlung möglich war, wurden zensiert

\section{Diskussion}

\section{Wartezeit auf ein Erstgespräch}

Die zu Beginn aufgestellte Hypothese, die durchschnittliche Wartezeit auf ein Erstgespräch habe sich durch die Reform verkürzt, konnte anhand der dokumentierten Zeiten aus den Psychotherapiepraxen nicht bestätigt werden. Wir waren davon ausgegangen, dass sich die Reform v.a. auf diese Wartezeit ausgewirkt haben gung (Bundespsychotherapeutenkammer 2018). Eine mögliche Erklärung wäre, dass einige der an der Studie teilnehmenden Praxen schwerpunktmäßig Krebspatienten mit psychischen Begleiterkrankungen behandeln und daher besonders schnell Termine anbieten müssen (Schulz et al. 2018). Allerdings wurde nicht erfasst, welche der Patienten krebserkrankt oder Angehörige von Krebserkrankten waren und welche nicht (auch bei Praxen mit psychoonkologischem Schwerpunkt sind nicht alle Patienten Krebsbetroffene). So konnten die Wartezeiten nur auf der Praxisebene verglichen werden, und hier fanden sich keine Belege dafür, dass diese Erklärung plausibel ist. Wir müssen also schlussfolgern, dass keine Anhaltspunkte dafür vorliegen, dass sich durch die Reform die Zeit zwischen Erstkontakt und Erstgespräch verringert hat.

\section{Wartezeit auf den Behandlungs- beginn}

Die zweite Annahme lautete, dass sich die Wartezeit auf den Beginn der eigentlichen Behandlung durch die Reform nicht verkürzt hat. Eine Verkürzung wäre zwar insofern zu erwarten gewesen, als durch die Einführung von (antragsfreien) Akutbehandlungen und den Wegfall der Gutachterpflicht für Anträge auf Kurzzeittherapien eine Reduktion bürokratischer Schritte und damit ein schnellerer Beginn von Therapien angedacht war (Gemeinsamer Bundesausschuss 2016, 2017). Auch die mit der Reform eingeführte Vermittlung durch Terminservicestellen und die Sprechstunden könnten dazu dienen, dass Patienten gezielter zu Therapeuten kommen und die Indikation schneller geklärt werden kann. Andererseits haben sich die Kapazitäten für Behandlungen dadurch nicht verändert allenfalls durch die Teilung von Kassensitzen. Deshalb waren wir davon ausgegangen, dass die gewünschte Verkürzung dieser Wartezeiten nicht wie erhofft eingetreten ist. Hier hat sich ein gemischtes Bild gezeigt. Im Durchschnitt hat sich die Wartezeit auf den Behandlungsbeginn in den im Rahmen dieser Studie untersuchten Praxen sogar um 2 Wochen verlängert. Der Anteil derjenigen, die vergleichsweise schnell, also innerhalb von 10 Wochen nach dem Erstkontakt, einen Behandlungs- 
Tab. 3 Anteil der Patienten, bei denen eine Behandlung in der eigenen Praxis begonnen wurde und Gründe für eine Nichtbehandlung

\begin{tabular}{|l|l|l|}
\hline & $n$ & $\%$ \\
\hline Behandlung in eigener Praxis & 663 & 43 \\
\hline Keine Behandlung, weil... & 885 & 57 \\
\hline Überweisung ins Krankenhaus & 7 & 0,5 \\
\hline keine Kapazität & 59 & 7 \\
\hline keine Indikation für ambulante Psychotherapie & 210 & 24 \\
\hline Patient lehnt Behandlung in Praxis ab & 100 & 11 \\
\hline Krisenintervention ausreichend & 89 & 10 \\
\hline Patient verstorben & 34 & 4 \\
\hline schlechter Allgemeinzustand des Patienten & 38 & 4 \\
\hline Verweis an andere Stelle (z. B. anderes Verfahren) & 55 & 6 \\
\hline unzureichende Therapiemotivation & 52 & 6 \\
\hline finanzielle Gründe & 12 & 1 \\
\hline kein weiterer Bedarf & 41 & 5 \\
\hline keine Passung & 22 & 2 \\
\hline andere Gründe & 24 & 3 \\
\hline Gründe nicht dokumentiert & 148 & 17 \\
\hline $\begin{array}{l}\text { Mehrfachnennungen möglich. Die Prozentwerte ab Zeile 3 der Tabelle beziehen sich auf die Patien- } \\
\text { ten, bei denen keine Behandlung zustande kam }(n=885)\end{array}$ & \\
\hline
\end{tabular}

platz bekamen, hat sich nach der Reform verringert (von $28 \%$ auf $16 \%$ ), aber der Anteil derjenigen, die sehr lange (mehr als 40 Wochen) auf einen Platz warten mussten, hat sich ebenfalls - allerdings nur minimal - verringert (von 5\% auf $4 \%)$. Dies könnte damit zusammenhängen, dass Psychotherapeuten durch die Einführung der verpflichtenden Sprechstunde (100 min/Woche bei ganzem Kassensitz) kapazitätsbedingt weniger Psychotherapieplätze vergeben können.

\section{Limitationen der Studie}

Begrenzt ist die Aussagekraft der durchgeführten Analysen dadurch, dass keine Angaben darüber vorlagen, ob und wie schnell die Patienten mit einer Psychotherapieindikation, die nicht in dieser Praxis behandelt werden konnten oder wollten, bei anderen Therapeuten eine Psychotherapie erhielten. Es kann also nur eine Aussage dazu getroffen werden, wie zügig die Behandlung innerhalb einer Praxis beginnen konnte. Eine weitere Einschränkung besteht darin, dass die Praxen natürlich nur dann eine Akte anlegten, wenn sie eine Leistung erbracht haben, also wenn zumindest ein Erstgespräch stattgefunden hat. Dadurch haben alle der in diese Studie eingeschlossenen Patienten ein Erstge-
Daten für vergleichbare Studien extrahiert werden.

\section{Stärken der Studie}

Stärken der Analysen bestehen darin, dass sie nicht auf Einschätzungen von Psychotherapeuten beruhen, die einem RecallBias unterliegen können, sondern auf dokumentierten Daten, und dass sowohlärztliche als auch psychologische und Kinder- und Jugendlichenpsychotherapeuten beteiligt waren. Es wäre wünschenswert, wenn weitere Praxen entsprechende Daten zur Verfügung stellen könnten, um mögliche Effektmodifikationen besser untersuchen zu können. So ist beispielsweise denkbar, dass die Reform nur bei bestimmten Praxistypen oder bei bestimmten Patientengruppen einen Effekt hatte. Dafür wäre jedoch eine breitere Datenbasis sinnvoll.

\section{Repräsentativität der Studien- teilnehmer} Patienten, die bei einem Therapeuten um ein Erstgespräch anfragen, dieses auch bekommen. Das ist bekanntlich nicht der Fall (Goldner et al. 2011), und so sollten die erhobenen Daten nicht interpretiert werden. Ebenfalls unbekannt ist, wie lange die Patienten selbst ggf. zögerten, bevor sie Kontakt mit der Praxis aufnahmen. Es ist denkbar, dass diese "Wartezeit" durch die Einführung von Terminservicestellen reduziert werden konnte. Allerdings kann dies nur durch Befragungen von Patienten geklärt werden, da die Therapeuten diese Informationen naturgemäß nicht haben. Eine Studie des Universitätsklinikums UIm zeigte, dass die Intensität des Suchens bei den Patienten unterschiedlich ist - manche führten bis zu 50 Telefonate mit potenziellen Therapeuten, manche nur eines (Von Wietersheim et al. 2021).

Bei der Planung der Datenerfassung hatten wir leider nicht bedacht, auch die Zugangswege in die Praxis zu erfassen, z. B. ob dies über eine Terminservicestelle erfolgte. Das wäre eine interessante $\mathrm{Zu}$ satzinformation gewesen. Deshalb möchten wir anregen, dass erstens Therapeuten diese Information in ihren Patientenakten dokumentieren, und zweitens, dass diese
Es ist die Frage zu stellen, inwiefern die teilnehmenden Patienten repräsentativ für alle in Psychotherapiepraxen vorstellig werdenden Personen sind. Dazu können Vergleiche mit anderen Studien dienen. Die in der repräsentativen Studie von Albani et al. (2010) befragten Psychotherapiepatienten waren bezüglich der Geschlechterverteilung sehr ähnlich (Anteil der Männer 28\% in der Studie von Albani et al. 2010; $25 \%$ in der vorliegenden Studie). Bezüglich des Alters gibt es Unterschiede, weil in die vorliegende Analyse auch Kinder und Jugendliche eingeschlossen wurden, dies war in der Studie von Albani et al. (2010) nicht der Fall. Hinsichtlich des Bildungsstatus sind beide Gruppen vergleichbar, wobei in der vorliegenden Studie der Anteil der Patienten mit Abitur etwas geringer war (34\% vs. $44 \%$ in der Studie von Albani et al. 2010). Allerdings kann auch dies mit der Teilnahme von Kindern und Jugendlichen an der vorliegenden Studie zusammenhängen. Es können keine Vergleiche hinsichtlich der F-Diagnosen angestellt werden. Diese konnten Albani et al. in ihrer Studie (2010) - naheliegenderweise - nicht erfassen, da es sich um Selbstauskünfte der Patienten handelte. Hierfür können stattdessen Abrechnungsdaten herangezogen 
werden: Laut einer Analyse von 9,7 Mio. Abrechnungsziffern für psychotherapeutische Leistungen (Gaebel et al. 2016) entfielen die meisten auf F3- und F4-Diagnosen (mit 36 und 39\%), gefolgt von F5 (8\%), F1 (7\%) und F2 (5\%). In dieser Analyse waren die F6- bis F9-Diagnosen nicht mit ausgewertet worden, sodass ein direkter Vergleich der Prozentangaben schwierig ist, aber eine Rangfolge ist ersichtlich. Demnach sind in der vorliegenden Befragung F4-Diagnosen häufiger vertreten als üblich. Dies könnte dann zu einem Bias geführt haben, wenn die Reform bei Patienten mit unterschiedlichen F-Diagnosen unterschiedlich gewirkt hätte.

\section{Resümee}

Die in den Praxen dokumentierten Daten liefern keine Anhaltspunkte dafür, dass sich die Wartezeit auf Erstgespräche bei Psychotherapeuten durch die Reform verkürzt hat. Das Warten auf einen Behandlungsplatz scheint nach der Reform sogar insgesamt etwas länger geworden zu sein, wobei die Anteile der sehr zügigen und der sehr späten Behandlungsbeginne etwas zurückgegangen sind.

\section{Fazit für die Praxis}

- Die Psychotherapiestrukturreform im Jahr 2017 hatte das Ziel, die Wartezeiten auf eine psychotherapeutische Behandlung zu verkürzen. Daten von 1548 Patientenakten aus 9 Praxen in 7 Bundesländern geben keine Anhaltspunkte dafür, dass dieses Ziel erreicht wurde.

- Die Zeit zwischen einer Kontaktaufnahme mit der Praxis und dem Erstgespräch betrug vor und nach der Reform im Durchschnitt jeweils 3 Wochen. Die durchschnittliche Zeit zwischen einer Kontaktaufnahme und dem Behandlungsbeginn war nach der Reform mit 20 Wochen sogar 2 Wochen länger als vor der Reform.

- Um die Wartezeiten auf einen Psychotherapieplatz effektiv verkürzen zu können, müssen weitere Maßnahmen erarbeitet und ergriffen werden.

\section{Waiting times for psychotherapy before and after the reform of the psychotherapy law in Germany}

Background and research question: One objective of reforming the German psychotherapy law in 2017 was to reduce the time patients wait until they can start psychotherapy. This study examined whether this goal has been achieved.

Methods: Psychotherapy offices in seven federal states in Germany extracted the dates of the first contact, first visit, and start of treatment from their files. Waiting times for a first visit and beginning treatment were compared using Kaplan-Meier curves and stratified log-rank tests.

Results: Data of 1548 patient records from 9 offices were extracted. The average waiting time for a first visit was 3 weeks both before and after the reform $(p=0.20)$. The time between the first contact and start of treatment was 18 weeks before and 20 weeks after the reform, whereby it was the very short waiting times ( $<10$ weeks) that decreased in particular $(p=0.0004)$.

Discussion: The data documented in the offices provide no evidence for a reduction in the waiting time for a first visit. Waiting times for beginning psychotherapy seem to have increased. Therefore, there is so far no indication that mental health care was improved in this respect by the reform.

\section{Keywords}

First interview · Treatment start · Treatment delay · Health care system · Health care utilization

\section{Korrespondenzadresse}

\section{Prof. Dr. Susanne Singer}

Abteilung Epidemiologie und Versorgungsforschung, Institut für Medizinische Biometrie, Epidemiologie und Informatik (IMBEI), Universitätsmedizin, Johannes GutenbergUniversität

Obere Zahlbacher Str. 69, 55131 Mainz, Deutschland

singers@uni-mainz.de

Förderung. Die Studie wurde gefördert durch den Innovationsfonds des Gemeinsamen Bundesausschusses (\# 01VSF19003).

Funding. Open Access funding enabled and organized by Projekt DEAL.

\section{Einhaltung ethischer Richtlinien}

Interessenkonflikt. S. Singer, L. Maier, A. Paserat, K. Lang, B. Wirp, J. Kobes, U. Porsch, M. Mittag, G. Toenges und D. Engesser geben an, dass kein Interessenkonflikt besteht.

Die Studie wurde von der zuständigen Ethikkommission der Landesärztekammer Rheinland-Pfalz begutachtet und befürwortet (\# 2020-14898).

Open Access. Dieser Artikel wird unter der Creative Commons Namensnennung 4.0 International Lizenz veröffentlicht, welche die Nutzung, Vervielfältigung, Bearbeitung, Verbreitung und Wiedergabe in jeglichem Medium und Format erlaubt, sofern Sie den/die ursprünglichen Autor(en) und die Quelle ordnungsgemäßnennen, einen Link zur Creative Commons Lizenz beifügen und angeben, ob Änderungen vorgenommen wurden.

Die in diesem Artikel enthaltenen Bilder und sonstiges Drittmaterial unterliegen ebenfalls der genannten Creative Commons Lizenz, sofern sich aus der Abbildungslegende nichts anderes ergibt. Sofern das betreffende Material nicht unter der genannten Creative Commons Lizenz steht und die betreffende Handlung nicht nach gesetzlichen Vorschriften erlaubt ist, ist für die oben aufgeführten Weiterverwendungen des Materials die Einwilligung des jeweiligen Rechteinhabers einzuholen.

Weitere Details zur Lizenz entnehmen Sie bitte der Lizenzinformation auf http://creativecommons.org/ licenses/by/4.0/deed.de.

\section{Literatur}

Albani C, Blaser G, Geyer M et al (2010) Ambulante Psychotherapie in Deutschland aus Sicht der Patienten. Teil 1: Versorgungssituation. Psychotherapeut 55:503-514

Bridler R, Orosz A, Cattapan K et al (2013) In need of psychiatric help-leave a message after the beep. Psychopathology 46:201-205

Bundesausschuss G (2016) Tragende Gründe zum Beschluss des Gemeinsamen Bundesausschusses über eine Änderung der PsychotherapieRichtlinie: Strukturreform der ambulanten Psychotherapie. G-BA, Berlin

Bundespsychotherapeutenkammer (2018) Ein Jahr nach der Reform der Psychotherapie-Richtlinie. Wartezeiten 2018. BPtK, Berlin

GaebelW,ZielasekJ, KowitzS(2016) Inanspruchnahme ambulanter psychotherapeutischer Versorgung Eine Analyse von Sekundärdaten. Nervenarzt 87:1201-1210

Gesundheit BF (2017) Bekanntmachung des Gemeinsamen Bundesausschusses über die Richtlinie über die Durchführung der Psychotherapie (Psy- 
chotherapie-Richtlinie) nach der Strukturreform. Bundesanz AT B3:1-16

Goldner EM, Jones W, Fang ML (2011) Access to and waiting time for psychiatrist services in a Canadian Urban area: a study in real time. Can J Psychiatry 56:474-480

Kruse J, Larisch A, Hofmann M et al (2013) Ambulante psychosomatische und psychotherapeutische Versorgung in Deutschland: Versorgungsprofile abgebildet durch Daten der Kassenärztlichen Bundesvereinigung (KBV). Z Psychosom Med Psychother 59:254-272

Schulz H, Bleich C, Bokemeyer C et al (2018) Psychoonkologische Versorgung in Deutschland: Bundesweite Bestandsaufnahme und Analyse. Wissenschaftliches Gutachten im Auftrag des Bundesministeriums für Gesundheit. Universitätsklinikum Hamburg-Eppendorf, Hamburg

Singer S, Beckerle J, Kleining B et al (2017) "Die Strukturen müssen viel flexibler sein“. Qualitative Befragung niedergelassener Psychotherapeuten zur Versorgung von Krebspatienten. Psychotherapeut 62:440-449

Von Wietersheim J, Seitz B, Rottler E et al (2021)

Aufwand, Erfolg und Scheitern bei der Suche nach einem ambulanten Psychotherapieplatz Ergebnisse einer prospektiven Studie. Gesundheitswesen 83:40-46

Wiegand HF, Saam J, Marschall U et al (2020)

Challenges in the transition from in-patient to out-patient treatment in depression. An analysis of administrative health care data from a large German health insurer. Dtsch Arztebl Int 117:472-479
Williams ME, Latta J, Conversano P (2008) Eliminating the wait for mental health services. J Behav Health Serv Res 35:107-114

Zepf S, Mengele U, Hartmann S (2003) Zum Stand der ambulanten psychotherapeutischen Versorgung der Erwachsenen in der Bundesrepublik Deutschland. Psychother Psychosom Medizinische Psychol 53:152-162

\section{Hier steht eine Anzeige.}

\section{Springer}


Hier steht eine Anzeige.

黑 Springer 